\title{
GEO 600 triple pendulum suspension system: Seismic isolation and control
}

\author{
M. V. Plissi, ${ }^{\text {a) }}$ C. I. Torrie, M. E. Husman, N. A. Robertson, K. A. Strain, and H. Ward \\ Department of Physics and Astronomy, University of Glasgow, Glasgow G12 8QQ, Scotland, \\ United Kingdom \\ H. Lück \\ Universität Hannover, Institut für Atom- und Molekülphysik, Abteilung Spektroskopie, \\ D-30167 Hannover, Germany \\ J. Hough \\ Department of Physics and Astronomy, University of Glasgow, Glasgow G12 8QQ, Scotland, \\ United Kingdom
}

(Received 28 September 1999; accepted for publication 21 February 2000)

In this article we describe aspects of the suspension system for each of the main optics (test masses) in the GEO 600 interferometric gravitational wave detector currently under construction in Germany. In particular we describe the triple pendulum, which is a key aspect of the overall system, discuss the details of the mechanical design, present transfer functions of the isolation performance, and display examples of impulse responses for the local control used to damp the low frequency modes of the triple pendulum. This local control is achieved using co-located sensing and feedback at the highest mass of the triple pendulum, providing a significant attenuation of local control noise to the fused silica test mass, the lowest mass of the triple pendulum. In order to enhance the vertical isolation, the triple pendulum will incorporate two stages of cantilever springs, and fused silica fibers will be used in the lowest pendulum stage in order to minimize thermal noise from the pendulum modes. It is expected that the thermal noise associated with the internal modes of the fused silica test mass (mass $\sim 6 \mathrm{~kg}$ ) will set the sensitivity limit for GEO 600 from 50 to $\sim 200 \mathrm{~Hz}$. The measured performance from individual stages of the prototype suspension system indicates that a seismic noise level which is a factor $\sim 3$ lower than this thermal noise level at $50 \mathrm{~Hz}$ can be achieved. (C) 2000 American Institute of Physics. [S0034-6748(00)02606-X]

\section{INTRODUCTION}

In an earlier article we described the revised specification for the GEO 600 (German-British) detector. ${ }^{1}$ The description explicitly stated that the system should not be limited by seismic noise above $50 \mathrm{~Hz}$. This figure was originally $100 \mathrm{~Hz}$. The decision to extend the detector sensitivity down to this lower frequency was motivated by studies ${ }^{2}$ of potential sources, e.g., the Crab pulsar, which are expected to emit gravitational waves in this frequency region. The sensitivity limit from thermal noise at $50 \mathrm{~Hz}$ is expected to be 2 $\times 10^{-22} / \sqrt{\mathrm{Hz}}$ which, with the GEO 600 optical scheme, corresponds to a test mass motion of $\left(7 \times 10^{-20} \mathrm{~m} /\right.$ $\sqrt{\mathrm{Hz}})_{50 \mathrm{~Hz}}$. The design objective for this seismic isolation system was to achieve a seismic noise level at each test mass a factor of 10 lower than this. To this end a triple pendulum suspension for the optics in the GEO 600 detector has been developed. The design has grown from initial work on double pendulum suspensions, used in both the Glasgow 10 $\mathrm{m}$ prototype detector ${ }^{3}$ and the mode cleaner mirrors in the GEO 600 detector. In order to enhance the vertical isolation, required for the main optics in the detector, a third pendulum stage was required. For some of the main optics, it is re-

\footnotetext{
a) Author to whom correspondence should be addressed; electronic mail: m.plissi@physics.gla.ac.uk
}

quired to apply global control forces in order to maintain the output of the interferometer on a dark fringe. In these cases a reaction pendulum is included so that these forces can be applied from a seismically isolated platform. In our earlier design ${ }^{1}$ the main double pendulum and reaction double pendulum were separately suspended from a common upper mass which was itself suspended. This proved to be a rather complex system both in terms of mechanical design and local control. For simplicity of design and construction the use of separate triple pendulums has now been adopted.

This article describes the design and testing of elements of the seismic isolation system with particular emphasis on the triple pendulum suspension. It also includes a description of the novel local control damping scheme for the triple pendulum suspension. Passive damping schemes for triple pendulums have been proposed; ${ }^{4}$ however, electronic feedback damping of the pendulum modes, properly executed, more easily avoids a noise penalty in the gravitational wave signal band.

In a companion article ${ }^{5}$ we discuss the modeling of the triple pendulum and consider the levels of cross coupling between the various degrees of freedom of the pendulum. This latter point is important when considering the requirement for the vertical isolation. 


\section{SEISMIC ISOLATION}

We aim to achieve the overall seismic isolation requirements with a combination of several elements, namely a two layer seismic isolation stack consisting of an active and a passive stage and a triple pendulum suspension incorporating two stages of soft vertical springs. The intermediate and test masses of the triple pendulum are made from fused silica with fused silica fibers between them in order to minimize thermal noise from the pendulum modes. It should be noted that the fused silica test mass $(5.6 \mathrm{~kg})$ used in the GEO 600 suspension described in this article is a factor of $\sim 2.5$ lighter than that previously proposed.

\section{A. Stack design}

The stack, which provides both horizontal and vertical isolation, consists of three legs, each consisting of two layers. The active lower layer will be discussed in further detail in Sec. III. It should be noted that in the present design the active isolation is not considered to contribute to the overall isolation in the gravitational wave detection band. The passive upper layer, which behaves like a mass on a damped spring, consists of a stainless steel block on pieces of graphite-loaded silicon rubber (RTV 615). These stacks are encapsulated in stainless steel bellows to prevent potential contamination of the optics of the interferometer by outgassing from the rubber elements. A fuller description of the design principles is outlined in the earlier article. ${ }^{1}$ The quality factor of the passive stage is chosen to be small ( $Q$ $\sim 10$ ) so as to reduce the amplitude of the seismic noise coupled at the resonances of the stack. Experiments at Glasgow have shown an attenuation of $\sim 20 \mathrm{~dB}$ in the vertical dimension and $\sim 30 \mathrm{~dB}$ in the horizontal dimension, at 50 $\mathrm{Hz}$, can be achieved with this single passive layer. Because the bellows are comparatively stiff in rotation and to reduce the possibility of transmission of rotational ground noise to the top of the stack, a rotational flexure is included at the top of each stack leg. Connecting the tops of the three stack legs together is a hexagonal box-section steel structure referred to as a stack stabilizer. This provides static stability to the entire suspension assembly. A further structure, the rotational stage, provides an interface between the stack stabilizer and the pendulum system. This rotational stage provides a primary level of rotational alignment for the pendulum assembly. In order to minimize friction, the bearing between the stack stabilizer and rotational stage consists of a sapphire disk sliding upon a highly polished ceramic thrust plate. Figure 1 is a schematic of the overall main suspension showing the stack and the triple pendulum suspension, including the two cantilever spring stages.

\section{B. Vertical isolation design elements}

The seismic isolation stages, both stacks and pendulums, have low horizontal resonant frequencies and therefore low transmissibilities in our working frequency band. However, in the vertical dimension the resonant frequencies are higher. This, together with the unavoidable cross coupling of vertical to horizontal motion of the test mass (a working value of $0.1 \%$ is used for our system), means that for practical isola-

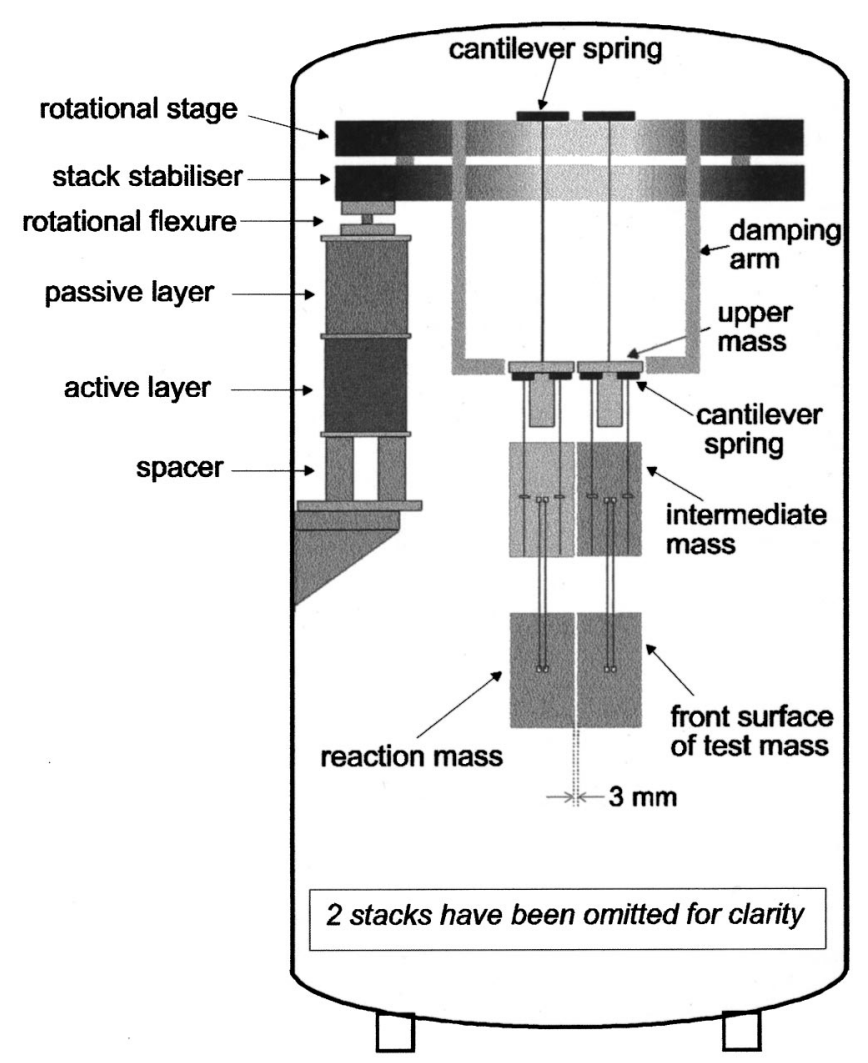

FIG. 1. Schematic of the overall main suspension (viewed perpendicular to the optic axis). A reaction pendulum, which is used to apply global feedback forces to the main pendulum, is shown adjacent to the main pendulum.

tion systems involving stacks and pendulums, isolation in the vertical dimension is likely to be the overall limiting factor in the total isolation achieved. Thus care has been taken in the design to ensure that enough vertical isolation is incorporated. In order to enhance the vertical isolation two additional spring stages are included. The first stage consists of two cantilever mounted blade springs supporting an upper mass. One end of each blade is fixed onto the rotational stage and from the tapered ends wires suspend the upper mass. From the upper mass a double pendulum is suspended by four cantilever blades (see Fig. 1). The springs are constructed from maraging (precipitation hardened) steel and have a nearly trapezoidal blade geometry. The design of the blades has been adapted from designs used in the FrenchItalian VIRGO project. ${ }^{6}$

The spring constant of a blade spring is given by ${ }^{7}$

$$
k=\frac{E a h^{3}}{4 l^{3} \alpha},
$$

where $l$ is the length of the blade, $a$ is the width of the blade base (at the clamp), $h$ is the blade thickness, $E$ is the Young's Modulus (for maraging steel $E \cong 190 \times 10^{9} \mathrm{~Pa}$ ), ${ }^{8}$ and $\alpha$ is a shape factor which is related to the ratio between the short end of the blade and its base $(\alpha=1.0$ for a rectangular shape and $\alpha=1.5$ for a triangle. For our blade designs it takes values between 1.36 and 1.44).

For the GEO 600 suspension, the final choices of blade parameters are as follows: The longer upper set of blades has the following dimensions: $l=240 \mathrm{~mm}, a=40 \mathrm{~mm}, h=2 \mathrm{~mm}$, 
and has an uncoupled ${ }^{9}$ vertical frequency of $\sim 2.7 \mathrm{~Hz}$. The shorter set of four blades, which supports the double pendulum from the upper mass, has dimensions: $l=124 \mathrm{~mm}, a=28$ $\mathrm{mm}, h=1 \mathrm{~mm}$, and has an uncoupled vertical frequency of $\sim 3 \mathrm{~Hz}$.

The choice of frequency for the cantilever stages is guided by our need to obtain a sufficiently good level of vertical isolation at our target frequency, while at the same time limiting the potential for long term creep. In our calculation we limit the maximum surface stress permissible at the support point to $\sim 800 \mathrm{MPa}$, approximately $50 \%$ of the elastic limit for Marval 18 maraging steel.

As we discuss in Sec. IV the design of the cantilever blades is complicated by the fact that they are not massless springs. This fact, as we will show, had a strong effect on the final choice of blade parameters.

\section{Triple pendulum}

As we have stated earlier, in order to satisfy our noise requirements for GEO 600 we require two additional vertical spring stages. From the outset there exist several constraints for the triple pendulum suspension which have to be taken into account before finalizing the design and calculating the mode frequencies. First, the intermediate and test mass main optics will be made from fused silica with four vertical fibers of fused silica between them, this design having been chosen to minimize the effects of thermal noise. ${ }^{10}$ Since we must not compromise the thermal noise performance of the suspension, steel cantilever blades cannot be placed between these masses, thus necessitating a triple pendulum design. Second, the low frequency resonant modes of the triple pendulum require damping in a way that does not introduce excess noise at the test mass. The application of active damping at the upper mass ensures that any extra motion caused by electronic noise in the feedback system is filtered by the double pendulum below before reaching the test mass. The control of the pendulum is outlined in more detail in Sec. III.

To facilitate feedback control of the pendulum system from a single mass, good coupling of the modes of the triple pendulum is necessary; in practice this requires that the masses and lengths of each stage are similar.

\section{CONTROL ISSUES}

\section{A. Active control}

In the active layer feed forward is used to control the effects of the micro-seismic peak around $0.2 \mathrm{~Hz}$ and feedback is used to improve the isolation at frequencies up to $\sim 10 \mathrm{~Hz}$. The active layer uses three degrees of freedom piezo actuators and geophones in a co-located system. This combination will reduce the bandwidth required in the damping servos and ease the acquisition of locking on the interferometer dark fringe.

\section{B. Local control}

Control systems are needed to damp the vibrational motion of mirror suspensions, reducing the mirror velocities to levels where interferometer signals, derived from the optical

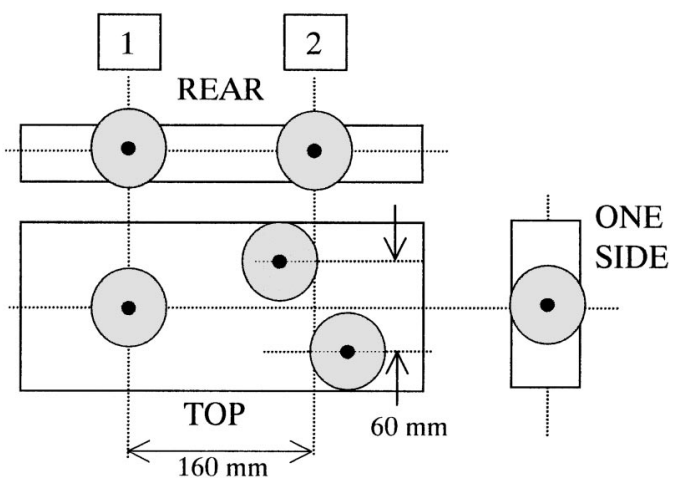

FIG. 2. Schematic view of the upper mass showing the locations of the actuator coils with respect to the mass. The two coils (numbered 1 and 2) acting on the rear of the mass control the longitudinal and rotational modes, three coils acting on the top control tilt, vertical and roll, and the one on the side controls the sideways motion. The coils are rigidly attached to the damping arm (see Fig. 1).

system formed by several suspended mirrors, become slow and linear enough to be used to control the mirror positions. ${ }^{11}$ The local controls serve this function by actively damping ${ }^{12}$ the most significant pendulum modes (frequencies of a few $\mathrm{Hz}$ ). These modes are typically excited by seismic noise and to keep the resulting motion down to the order of a wavelength the $Q$ should be $\leqslant 5$. Other requirements are minimum additional noise at the mirror and a robust system that is tolerant of the effects of small constructional errors.

The local control system for the GEO 600 triple pendulum suspension uses simple optical shadow sensors ${ }^{3}$ to sense the position of the upper mass with respect to a fixed rigid damping arm extending from the rotational stage. In these sensors an infrared beam from a light emitting diode (LED) is partially interrupted by an opaque "flag" affixed to the moving mass. The beam is detected at a $\mathrm{Si}$ photodiode and the resulting current is approximately proportional to the penetration distance of the flag into the beam. Typical ranges are $>1 \mathrm{~mm}$ and noise levels are $\sim 10^{10} \mathrm{~m} / \sqrt{\mathrm{Hz}}$ for simple and easily affordable devices. The simplest and most robust controller design is then obtained if the sensors are colocated with the actuators (the sensors measure a displacement of a point on which the actuators provide a force). Since it is hard to make low noise wide range sensors and since co-location is desired, the sensors and actuators are placed as high on the pendulum as possible (acting on the upper mass). Six such actuators are needed, arranged so that they couple to six degrees of freedom of the mass on which they act. The arrangement of actuators is as shown in Fig. 2. If the further step of using similar control laws (apart perhaps for a gain factor) for all six controllers is taken, then the system will have good tolerance to the effect of constructional errors.

A fixed electromagnet acting on a permanent magnet bonded to the mass forms the actuator. Approximately 1100 turns on the (right circular) coil are used giving a coil resistance of around $50 \Omega$ at room temperature. A coil length of 8 $\mathrm{mm}$ and a magnet length of $10 \mathrm{~mm}$ have been chosen in order to achieve the desired force-distance profile. Selecting a magnet with a diameter of $10 \mathrm{~mm}$ constrained the coil to 

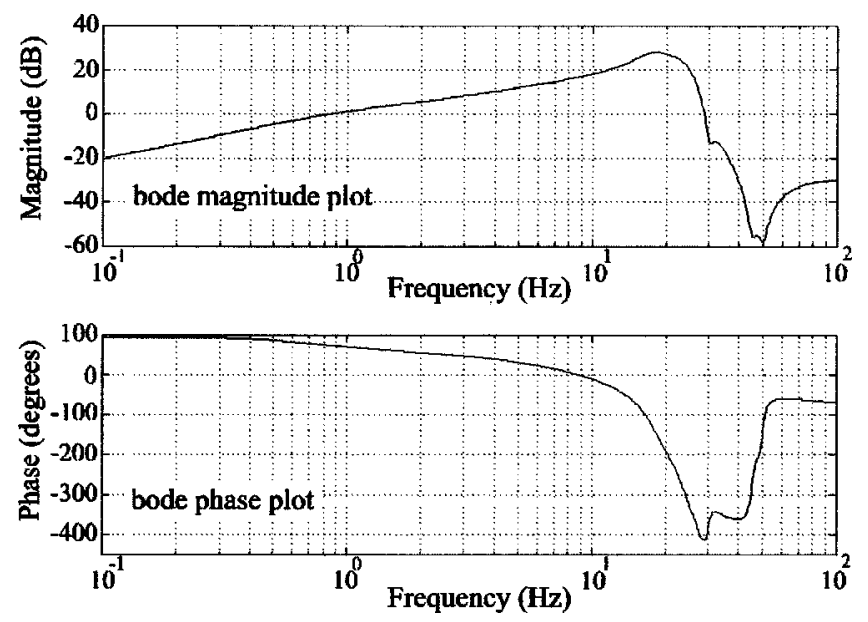

FIG. 3. Bode magnitude and phase plots illustrating the open loop response of the controller. The controller contains a high pass corner filter $0.7 \mathrm{~Hz}$; transitional differentiator $0.35-0.8 \mathrm{~Hz}$; transitional differentiator $2-14 \mathrm{~Hz}$; two pole low pass $18 \mathrm{~Hz}, Q=3$, notch $30 \mathrm{~Hz}$; two pole low pass $24 \mathrm{~Hz}$, $Q=4$, notch- $45 \mathrm{~Hz}$; two pole low pass $28 \mathrm{~Hz}, Q=5$, notch $=50 \mathrm{~Hz}$ and a simple low pass at $14 \mathrm{~Hz}$.

have inner and outer diameters of 28 and $50 \mathrm{~mm}$, respectively (allowing for clearance, glass encapsulation, and coil former). With the Nd:Fe:B ceramic magnetic, a peak force of $\geqslant 0.1 \mathrm{~N}$ is obtained. The current range used, $I_{\max } \leqslant 100 \mathrm{~mA}$ is chosen to give low noise, $\leqslant 40 \mathrm{pA} / \sqrt{\mathrm{Hz}} \mathrm{rms}$, at $50 \mathrm{~Hz}$ and above. A satisfactory design of local control system will ensure good damping performance over the range of frequencies occupied by the low frequency pendulum modes (approximately $0.4-5 \mathrm{~Hz}$ for our system), together with negligible noise penalty in the gravitational wave signal band. Both of these requirements place significant constraints on the servo design: on the one hand sufficient electronic phase lead must be provided at the resonances to produce optimum damping, while on the other hand, above the local control bandwidth the feedback sensor noise must be strongly attenuated.

Simple "velocity" feedback would fail to meet these requirements. Our servo design consists of a multistage filter consisting of simple and transitional differentiator stages to achieve phase lead, together with a combination of simple and resonant low pass filters for noise attenuation. The resonant Scultété filters that we use provide both minimum phase lag before the resonant peak and a notch in their response in the attenuating region. The former feature helps to retain adequate phase lead at the highest of the pendulum resonances to be damped, while the frequencies of the notches from the resonant filters can be tuned to optimize noise attenuation at the lower end of the gravitational wave band, and also to avoid unwanted excitation of the undamped high frequency pendulum modes involving extension of the suspending fibers (see Sec. IV). It should be noted that although the response of the Scultété filter does not fall continuously with frequency above the notch, the mechanical filtering action from the pendulums does continue and the attenuation provided by the overall transfer function is satisfactory. The transfer function of the controller is illustrated in Fig. 3.

The choice of local control action at the upper mass

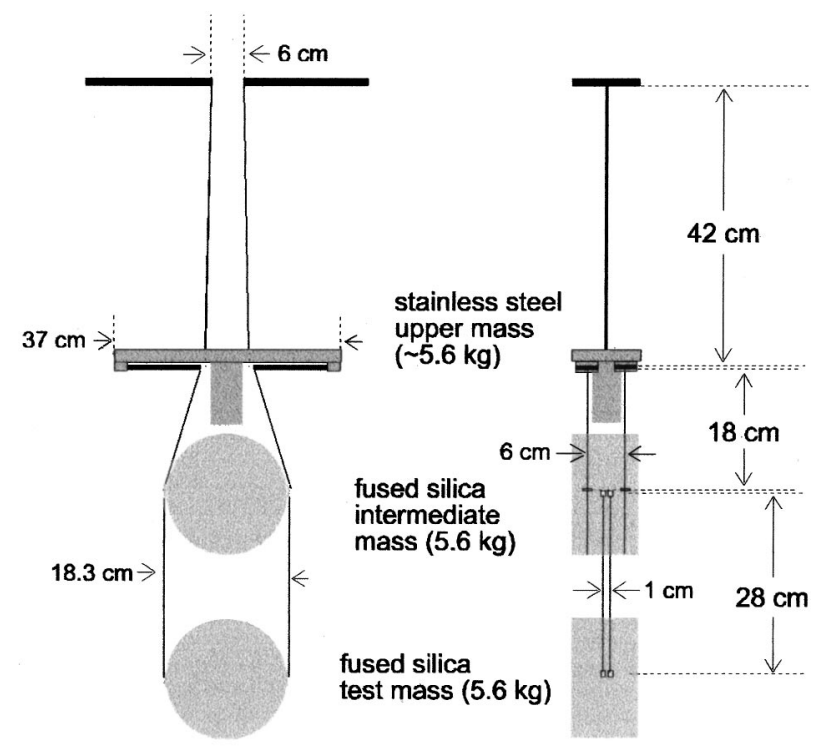

FACE VIEW

\section{SIDE VIEW}

FIG. 4. Mechanical parameters for the GEO 600 triple pendulum suspension. Other parameters not shown in the schematic are as follows: (with reference to the face view) the separation of the upper wires at the upper mass $=8 \mathrm{~cm}$, separation of the intermediate wires at the intermediate mass $=19.3 \mathrm{~cm}$. The high tensile steel upper and intermediate suspension wires have radii of 275 and $175 \mu \mathrm{m}$, respectively, and the lower fused silica suspension wires are expected to have a radius of $154 \mu \mathrm{m}$. The wires break off $1 \mathrm{~mm}$ above or below the center of mass of each of the three masses of the triple pendulum in the direction of increased static stability. the intermediate mass and test mass are both $10 \mathrm{~cm}$ thick and have a maximum diameter of $18 \mathrm{~cm}$.

places some constraints on pendulum design. It is necessary to ensure that every mode (to be damped) of the compound pendulum couples to motion along at least one of the sensed directions of the highest mass. This coupling can be made strong enough by following two design principles: masses and moments of inertia about corresponding axes of each pendulum stage should be similar (within a factor of approximately 2), and the stiffness of the passive restoring forces should increase slightly down the pendulum (again a factor of 2 from top to bottom is a starting point). These constraints have been found to be simple to incorporate into multiple pendulum suspension designs. The final choice of parameters chosen for the GEO 600 triple pendulum suspensions is illustrated in Fig. 4.

The design of the local controller and prediction of its behavior require a comprehensive dynamical model for the suspension, and this will be discussed in Sec. IV.

\section{Global control}

In addition to the local control actuation there is additional actuation providing three degrees of freedom between all masses of a reaction pendulum and the corresponding masses of a main pendulum. This is called global control actuation. At the lowest level of the suspension we propose to apply the feedback via an electrostatic drive system. At the intermediate level the feedback uses coil-magnet motors less powerful than those used at the upper mass for local control. 


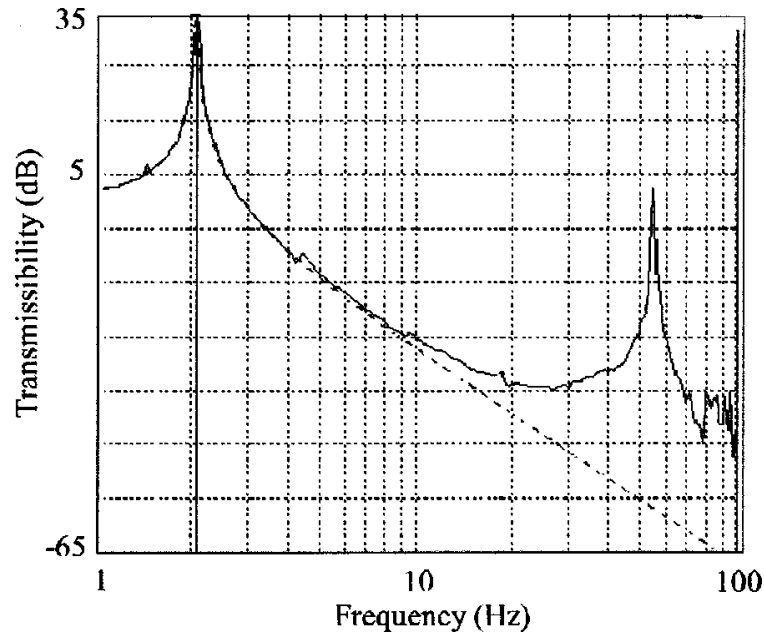

FIG. 5. Vertical transfer function of the prototype set of upper blade springs (illustrating nonideal behavior). The solid trace is the experimental transfer function and the dotted trace is the theoretical transfer function for an ideal massless spring with the same parameters. The first internal resonance is at $\sim 55 \mathrm{~Hz}$.

\section{TRANSFER FUNCTIONS OF A PROTOTYPE SUSPENSION SYSTEM}

A prototype triple pendulum was constructed in Glasgow. The intermediate and test masses were made from aluminum with the same mass and outer dimensions as the fused silica masses to be used in the GEO 600 suspensions. High tensile steel wires were used as the suspension wires. The purpose of this prototype was to test the performance of the blade springs, check that the pendulum mode frequencies matched with theory, and to test the performance of the damped triple pendulum in the longitudinal direction. The theoretical response of the uppermost stage, which is suspended from two cantilever blades, each having the following dimensions: $l=370 \mathrm{~mm}, a=82 \mathrm{~mm}, h=2 \mathrm{~mm}$, was obtained using MATLAB, ${ }^{13}$ and shows a falloff of $1 / f^{2}$ above the uncoupled frequency of the blade, (see Fig. 5). This simple model assumes that the blade acts as a massless spring. The measured vertical transfer function from the rotational stage to the upper mass of the triple pendulum, where the pendulum was driven by a shaker constructed from piezoelectric elements and the response measured using accelerometers, is also shown in Fig. 5. The flattening of the response above $\sim 20 \mathrm{~Hz}$ is consistent with measurements carried out by the VIRGO team on similar blades. ${ }^{14}$ The first internal mode at $55 \mathrm{~Hz}$ is also shown in the graph. The leveling out of the transfer function is due to the fact that the blades have a finite mass, and that the attachment point of the wires is not at the center of percussion of the blade.

\section{A. Mode frequencies}

The resonant frequencies were obtained by exciting the triple pendulum and using a spectrum analyzer to measure the response with an accelerometer. The experimental results for the prototype triple pendulum are given in Table I. The companion article ${ }^{5}$ compares the experimental results with those predicted from theory, and shows good agreement between experiment and theory.
TABLE I. Prototype mode frequencies (Hz). Approximate mode names are given as an indication of the motion. Longitudinal: longitudinal translation along the optic axis. Sideways: sideways translation transverse to the optic axis. Vertical: vertical translation. Tilt: tilt about the transverse axis. Rotation: rotation about the vertical axis. Roll: roll about the optic axis. The frequencies given in parentheses are predictions from theory. These modes were difficult to observe experimentally.

\begin{tabular}{cl}
\hline \hline \multicolumn{1}{c}{ Axis } & \multicolumn{1}{c}{ Experimental } \\
\hline Tilt/longitudinal: & $3.5,2.6,2.2$ \\
& $1.4,0.6,(0.5)$ \\
Roll/sideways: & $(52.0),(3.3),(2.5)$ \\
& $1.4,1.0,0.6$ \\
Rotational: & $3.1,1.6,(0.4)$ \\
Vertical: & $37.0,3.7,1.0$ \\
\hline \hline
\end{tabular}

Observe that the upper roll and vertical frequencies are comparatively high. These frequencies are associated with the extension of the wires in the lowest pendulum stage which, as we have noted, has no soft vertical springs.

\section{B. Step response}

The theoretical and experimental response of the two longitudinal actuators is shown in Fig. 6. A step signal was applied to both of the longitudinal actuators arranged along the long axis of the upper mass (see Fig. 2) and the response was measured with sensors affixed to this upper mass. The experimental plots fit closely with the theoretical prediction from a MATLAB model.

\section{TRANSFER FUNCTIONS OF THE FINALIZED SUSPENSION SYSTEM}

The upper blade has since been redesigned (see Sec. II B for the blade parameters) so that the first internal mode is above $100 \mathrm{~Hz}$. In addition, because the redesigned blade is shorter and lighter the transfer function more closely resembles an ideal blade (see Fig. 7), with a falloff around $1 / f^{2}$ to at least $\sim 30 \mathrm{~Hz}$. Although this blade design has an un-

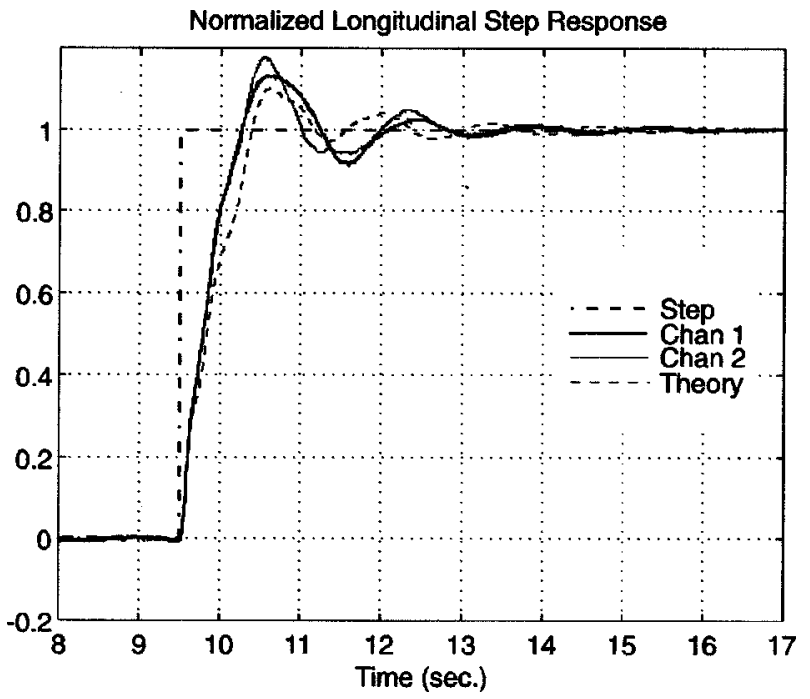

FIG. 6. Longitudinal step responses for the two longitudinal actuators (channels 1 and 2) compared to the theoretical prediction from a MATLAB model. 


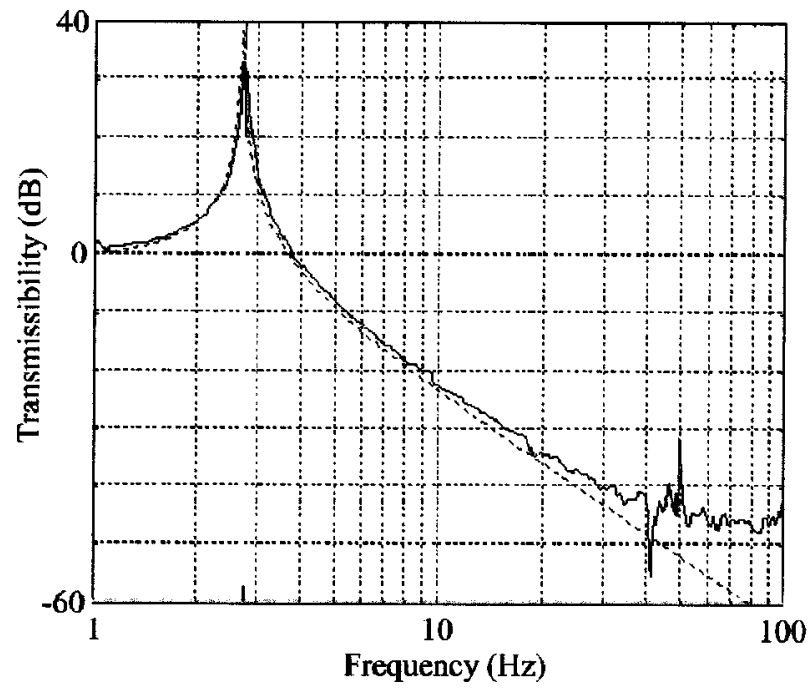

FIG. 7. Vertical transfer function of the redesigned set of upper blade springs (illustrating more closely ideal behavior). The solid trace is the experimental transfer function and the dotted trace is the theoretical transfer function for an ideal massless spring with the same blade parameters. The notch at $40 \mathrm{~Hz}$ is associated with a structural resonance in the measurement system. The peak near $50 \mathrm{~Hz}$ is due to electrical mains interference.

coupled resonant frequency higher than the initial one, because of the manifest nonideal behavior of the initial design, the overall performance of the new blade design is better at $50 \mathrm{~Hz}$. A transfer function of the lower blades was also obtained. An attenuation of $\sim 55 \mathrm{~dB}$ at $50 \mathrm{~Hz}$ was measured with the blades behaving ideally up to a frequency of $\sim 80$ $\mathrm{Hz}$. The first internal mode of the lower blades was measured at $220 \mathrm{~Hz}$. This internal mode will not be damped; due to the mechanical filtering action from the pendulum the attenuation provided by the overall transfer function is adequate at this frequency. It is intended to damp the internal modes of the upper blades using resonant dampers of a similar design to that developed by the VIRGO team. ${ }^{15}$

In Fig. 8 are shown the horizontal and vertical transfer functions of the triple pendulum generated by a model of the suspension. ${ }^{5}$ This model includes the flattening of the trans-

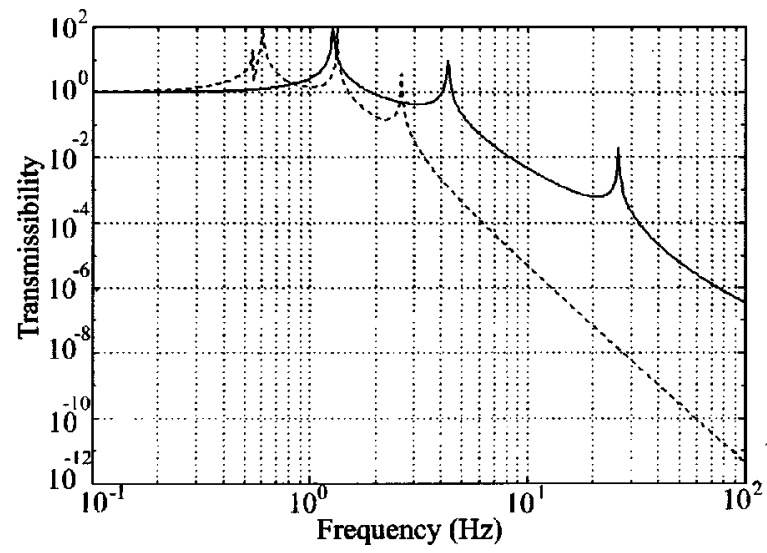

FIG. 8. Theoretical MATLAB plots of the vertical (solid trace) and horizontal (dotted trace) transfer functions for the GEO 600 triple pendulum suspension. the flattening of the vertical transfer function due to the nonideal nature of the cantilever blades is included in the model. The horizontal transfer function is calculated from the top point of the suspension to $1 \mathrm{~mm}$ above the center of mass of the test mass.

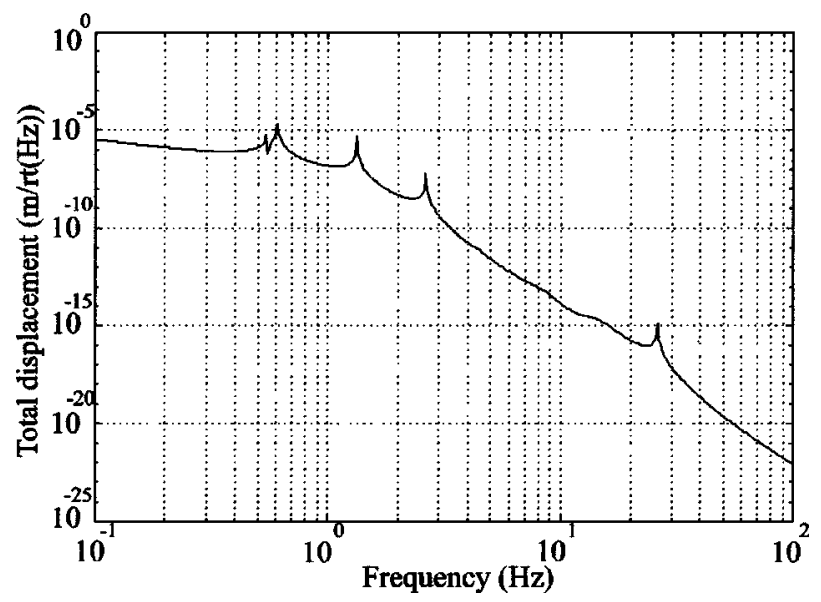

FIG. 9. A theoretical MATLAB plot of the total displacement noise of the test mass, which was calculated from transfer functions of triple pendulum and stack, in both vertical and horizontal dimensions, and incorporates a typical seismic noise input.

fer function due to the nonideal behavior of the blades. The extra peak around $0.5 \mathrm{~Hz}$ is due to tilt coupling into horizontal. Modeling of the local control servo indicates that all the modes, with the exception of the vertical and roll frequencies associated with the final stage, can be actively damped to a $Q$ value less than 5 . The highest frequency modes, which will lie between 20 and $\sim 40 \mathrm{~Hz}$ for the finalized GEO 600 design, couple only very weakly to the main interferometer signal, and are left undamped in the present setup as they occur too near the gravitational wave band to allow sufficient attenuation of electronic noise that would be associated with the use of a local sensor.

Figure 9 is a graph showing the total displacement noise of the test mass as a function of frequency. The plot was obtained as follows: first, the horizontal and vertical transfer functions were calculated individually. These included the stack, the triple pendulum, and a seismic noise input of $\left[10^{-7} / f^{2}\right] \mathrm{m} / \sqrt{\mathrm{Hz}}$, which is typical of the spectrum at the GEO 600 site in the region $20-300 \mathrm{~Hz} .{ }^{16}$ Also included was the flattening of the response due to the nonideal nature of the cantilever blades. Then the horizontal and vertical transfer functions were added in quadrature (incorporating a $0.1 \%$ cross coupling factor from vertical to horizonal motion) to obtain the final plot, which is dominated by vertical motion above $\sim 10 \mathrm{~Hz}$. The stack frequencies included in the calculation are measured uncoupled frequencies. This is a valid input since, although the individual masses of the triple pendulum are comparable and therefore well coupled, the dynamic mass loading on the stack is significantly higher, consisting of the steel mass block, stack stabilizer, and rotational stage, and the damping arms (see Fig. 1). This means that the stack can be taken, for the purposes of this calculation, to be uncoupled from the rest of the suspension. The coupled pendulum frequencies come from our theoretical model. The highest vertical frequency of the system, namely $26 \mathrm{~Hz}$, is associated with the lowest stage of the triple pendulum, which will use fused silica fibers with an assumed radius of $154 \mu \mathrm{m}$.

Therefore at $50 \mathrm{~Hz}$ we predict a resulting test mass mo- 
tion $d x \sim\left(2.4 \times 10^{-20} \mathrm{~m} / \sqrt{\mathrm{Hz}}\right)_{50 \mathrm{~Hz}}$. This corresponds to a seismic noise level, which is a factor of $\sim 3$ lower than the expected motion due to thermal noise associated with the internal modes. This is acceptable since the effect of adding this seismic noise level to the thermal noise would increase the overall noise level by only $\sim 5 \%$.

We should emphasize that the above calculations do not apply above $\sim 120 \mathrm{~Hz}$ due to the internal modes of the isolation system (cantilever blades, etc.) and the presence of wire resonances. However, since the isolation provided by the system in general increases as a steep function of frequency, at these high frequencies it should be more than satisfactory.

\section{ACKNOWLEDGMENTS}

The authors would like to thank our colleagues both in Glasgow and in Germany for their interest in this work. We would like to thank both Riccardo De Salvo (CALTECH) and Stefano Braccini (VIRGO Project Team) for their helpful advice on cantilever spring design. In addition we would like to thank the JILA workshop, Boulder, CO, for supplying the prototype cantilever blades for use in our experiments. The authors acknowledge the financial support of the University of Glasgow and the PPARC. K.A.S. was in receipt of a PPARC Advanced Fellowship.
${ }^{1}$ M. V. Plissi, K. A. Strain, C. I. Torrie, N. A. Robertson, S. Killbourn, S. Rowan, S. M. Twyford, H. Ward, K. D. Skeldon, and J. Hough, Rev. Sci. Instrum. 69, 3055 (1998).

${ }^{2}$ K. S. Thorne, Gravitational Waves, Proc. Snowmass 95 Study on Particle and Nuclear Astrophysics and Cosmology, edited by E. W. Kolb and R. Peccei (World Scientific, Singapore, 1995), p. 18.

${ }^{3}$ D. I. Robertson, E. Morrison, J. Hough, S. Killbourn, B. J. Meers, G. P. Newton, N. A. Robertson, K. A. Strain, and H. Ward, Rev. Sci. Instrum. 66, 4447 (1995).

${ }^{4}$ K. Tsubono, A. Araya, K. Kawabe, S. Moriwaki, and N. Mio, Rev. Sci. Instrum. 64, 2237 (1993).

${ }^{5}$ M. E. Husman, C. I. Torrie, M. V. Plissi, K. A. Strain, N. A. Robertson, and J. Hough, Rev. Sci. Instrum. 71, 2546 (2000).

${ }^{6}$ A. Giazotto et al., in First Edoardo Amaldi Conference on Gravitational Wave Experiments, edited by E. Coccia, G. Pizzella, and F. Ronga (World Scientific, Singapore, 1995), p. 86.

${ }^{7}$ R. Poggiani (private communication), the VIRGO project team.

${ }^{8}$ R. P. Wei, Ferrous Alloys, in Aerospace Structural Metal Handbook (Cindas, Purdue University, 1970).

${ }^{9}$ The frequency observed for a spring in a particular stage supporting only the mass of that stage.

${ }^{10}$ S. Rowan, S. M. Twyford, R. Hutchins, J. Kovalik, J. E. Logan, A. C. McLaren, N. A. Robertson, and J. Hough, Phys. Lett. A 233, 303 (1997).

${ }^{11}$ M. J. Lawrence, B. Willke, M. E. Husman, E. K. Gustafson, and R. L. Byer, J. Opt. Soc. Am. B 16, 523 (1999).

${ }^{12}$ P. J. Veitch, N. A. Robertson, C. A. Cantley, and J. Hough, Rev. Sci. Instrum. 64, 1330 (1993).

${ }^{13}$ MATLAB for Windows, copyright 1984-1994, The Mathworks Inc. Version 4.2b (1994)

${ }^{14} \mathrm{G}$. Cella and A. Viceré, Super Attenuator vertical performance beyond the low frequency range, VIRGO internal document (June 1997).

${ }^{15}$ S. Braccini, C. Bradaschina, R. Del Fabbro, A. Di Virgilio, I. Ferrante, F. Fidecaro, R. Flaminio, A. Gennai, A. Giassi, A. Giazotto, G. Gorini, G. Losurdo, F. Palla, A. Pasqualetti, D. Passuello, R. Poggiani, G. Torelli, and Z. Zhang, Rev. Sci. Instrum. 67, 2899 (1996).

${ }^{16} \mathrm{M}$. Pickenpack and B. Willke (private communication). 